\title{
Avaliação da Mortalidade de Grupos Indígenas do Norte do Paraná - 1990 a 1999
}

\author{
Evaluation of the Mortality of Indian Groups of the North of Paraná \\ State, 1990 to 1999
}

\author{
Stela Candioto Melchior \\ Departamento de Saúde Coletiva - Universidade Estadual de Londrina - PR
}

Darli Antonio Soares*

Departamento de Saúde Coletiva - Universidade Estadual de Londrina - PR

Selma Maffei de Andrade

Departamento de Saúde Coletiva - Universidade Estadual de Londrina - PR

Rosa Maria Kayoko Izumi

Administração Executiva Regional da Fundação Nacional do Índio - Londrina - PR

\begin{abstract}
Resumo
Com o objetivo de conhecer a situação de saúde de índios que vivem no norte do Paraná, realizou-se estudo descritivo, em que são analisados os seguintes indicadores: coeficiente de mortalidade infantil e seus componentes (mortalidade neonatal e pós-neonatal), indicador de Swaroop-Uemura e indicador de Guedes adaptado por Pereira, no periodo de 1990 a 1999. A análise foi feita, comparando dois períodos: 1990-94 e 1995-99, para evitar flutuações aleatórias devido ao número pequeno de casos. Os dados foram obtidos em livros de registros de nascimentos e óbitos dos índios, existentes em cada reserva indígena, bem como em declarações de nascidos vivos e declarações de óbitos quando esses eventos ocorreram em hospitais. Para evitar perdas ou duplicidade nas informações, esses dados foram ainda comparados com os relatórios enviados à Fundação Nacional do Índio (FUNAI). Os resultados revelaram queda do nível de saúde da população indígena dessa região, com piora de todos os indicadores e aumento da mortalidade prematura, o que sugere a piora das condições de vida e a necessidade de rever as estratégias nas ações ligadas à assistência à saúde desses povos.
\end{abstract}

\section{Palavras-Chave}

Indicadores de Saúde; Perfil de Saúde; Índios Sul-Americanos.

\section{Summary}

A descriptive study was conducted with the objective of evaluating the health status of indians who live in the north of the State of Paraná, Brazil. For the period of 1990 to 1999, the following health indicators were calculated and analyzed: infant mortality rate and its components (neonatal and post-neonatal mortality), Swaroop-Uemura indicator, and Guedes indicator (adapted by Pereira). Analysis was performed comparing two periods: 1990 to 1994 and 1995 to 1999 in order to avoid random variation due to the small number of cases. Data were obtained from books of births and deaths registration in each indigenous reserve, as well as from death and birth certificates when these events had happened in hospitals. Data was compared with the reports sent to the Indian National Foundation (FUNAI) to avoid duplicated and to recover omitted information. Results revealed a decline in the health status of the indigenous population of this region with an increase in premature mortality and a decline in all mortality indicators. These suggest deterioration of the living conditions and the need to revise strategies of health care actions related to this population.

Key Words

Health Indicators; Health Profile; South American Indians.

\footnotetext{
* Endereço para correspondência: Núcleo de Estudos em Saúde Coletiva (NESCO) - Av. Robert Koch, 60 Vila Operária - Londrina/PR. CEP: 86.028-350.
}

E-mail: soaresda@sercomtel.com.br 


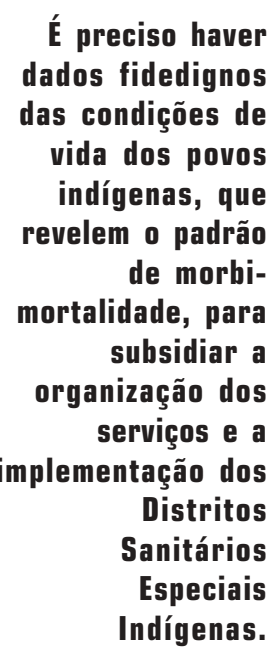

Informe Epidemiológico do SUS

\section{Introdução}

A saúde da população indígena no Brasil, após muitos anos sem uma política explícita, começa a ser discutida e analisada como parte da Política Nacional de Saúde. ${ }^{1}$ A necessidade de se trabalhar de forma integralizadora, que garanta o acesso dessa população aos serviços do Sistema Único de Saúde (SUS), não se confronta com a busca na elaboração de ações específicas que respeitem as características básicas que os individualizam e os diferenciam de outras populações.

Historicamente, os diversos grupos indígenas do Brasil sofreram uma grande perda populacional desde seu primeiro contato com os descobridores e, posteriormente, com os colonizadores, que constantemente os eliminavam durante o processo de ocupação do território brasileiro, além de expô-los a inúmeras doenças infecciosas até então desconhecidas ao seu sistema imunológico. Apesar da ausência de dados fidedignos sobre a população total indígena no início do século XVI, de acordo com a Fundação Nacional de Saúde (Funasa) existiam cerca de 5 milhões de pessoas. ${ }^{1}$ Atualmente, estima-se que essa população esteja restrita a aproximadamente $350 \mathrm{mil}$ pessoas. $^{2}$

Outras sucessivas perdas impostas aos grupos indígenas, como de sua língua nativa, de sua liberdade, de suas terras, de seus costumes e tradições, fizeram com que essa população sofresse também uma desestruturação social e perdesse muito de sua autonomia e auto-estima, tornando-a ainda mais vulnerável às diferentes epidemias. ${ }^{1}$

Atualmente, as comunidades indígenas vivem em áreas que, em muitos casos, não permitem a manutenção do seu antigo modo de viver, defrontando-se com problemas pela posse da terra e de devastação do ambiente natural, que comprometem a integridade de sua organização social e de sua própria saúde. ${ }^{1,3}$

O Estado do Paraná possui, atualmente, 17 terras indígenas (reservas que representam $0,3 \%$ da área originalmente ocupada), que se diferenciam entre si em tamanho de populações, áreas geográficas e etnias existentes. Em comum elas têm a falta de alimentos, a subnutrição, a precariedade e a inexistência de alternativas econômicas". ${ }^{3}$

As etnias que constituem as populações indígenas paranaenses são as caingangue, guarani e xetá. A maioria sobrevive da lavoura de subsistência, trabalho braçal em fazendas vizinhas das reservas e venda de artesanatos. ${ }^{3}$ As comunidades indígenas do norte do Paraná não fogem a esses problemas, vivem em cinco reservas que estão homologadas e demarcadas, o que não impede que ocorram invasões por outro grupo segregado da sociedade - os sem-terra. ${ }^{3}$

A acirrada competição pelas terras, resultante da busca pelo progresso de sociedades estranhas aos povos indígenas, fez com que os índios fossem "espremidos pelas frentes de expansão", o que resultou na ameaça de sua integridade. O caso dos xetá, etnia registrada apenas no Estado do Paraná, é exemplo marcante: descobertos no início dos anos 50 , foram rapidamente dizimados e contam atualmente com apenas seis integrantes, parentes entre si, o que compromete sua reprodução. ${ }^{3}$ Fato semelhante foi observado entre os latundê, do Estado de Rondônia, que possuem hoje apenas 37 pessoas.

A garantia de demarcação de terras indígenas é fator básico para que se garanta a produção e reprodução dos grupos indígenas, assim como sua integridade física, material e de sua tradições, fazendo com que cesse o processo de despopulação, e incremente a recuperação populacional. Esse fato já vem sendo observado no Estado do Paraná, que, no período de 1975 a 1997, registrou um aumento da população indígena - de cerca de 2.500 para aproximadamente $9.000 .^{3}$

Para que se preserve também a saúde dos povos indígenas, é necessário rever a forma de assistência prestada, considerando as diferenças culturais e epidemiológicas, além de incentivar que os próprios índios tenham parcela de 
participação no cuidado com seu povo e nas decisões que digam respeito a essa assistência. ${ }^{1}$ Os Distritos Sanitários Especiais Indígenas, que começaram a ser implantados no Brasil em 1999, contemplam essas características para a assistência aos povos indígenas e poderão ser instrumentos importantes para suprir as necessidades e especificidade desses povos. ${ }^{1}$

Para que esse serviço seja efetivo e possa melhorar o nível de saúde dos povos indígenas, é preciso que haja dados mais fidedignos das condições de vida dessa população, que revelem como está o crescimento desse povo, qual o seu padrão de morbi-mortalidade, enfim, dados que possam subsidiar ações concretas para uma melhor organização dos serviços e na própria implementação dos distritos nas terras indígenas. Esta pesquisa teve como objetivo, portanto, avaliar indicadores de mortalidade dos povos indígenas do norte do Paraná, no período de 1990 a 1999, e fornecer dados que possam contribuir para uma melhor estruturação da atenção à saúde dos índios que habitam essa região.

\section{Material e métodos}

Esta pesquisa abrangeu as cinco terras indígenas do norte do Paraná:

a) Apucaraninha, que pertence ao Município de Londrina, tendo uma área de 5.574ha, com uma população de 1.074 índios. Há três aldeias sediadas nessas terras (Sede, Barreiro e Toldo). É a reserva que possui a maior relação área por aldeia (1.858ha), porém também é a que possui o maior número de pessoas por aldeia $(n=358)$. Há predomínio de caingangues. Está localizada a $30 \mathrm{~km}$ do Município de Tamarana;

b) São Jerônimo, localizada no Município de São Jerônimo da Serra, possui 1.339 ha de área, com uma população de 251 índios, tendo uma única aldeia, a Sede. Com predomínio de guaranis, possui também um xetá e poucas famílias caingangue. Dista $3 \mathrm{~km}$ do Município de São Jerônimo; c) Barão de Antonina, pertencente também ao Município de São Jerônimo da Serra, com 3.571ha de área indígena e população de 400 índios. As aldeias ali localizadas são Sede e Cedro, portanto, com 1.785 ha e 200 pessoas por aldeia, em média. Há predomínio de caingangues, tendo também algumas famílias guaranis. As aldeias ficam a $18 \mathrm{~km}$ da cidade;

d) Laranjinha, pertence ao Município de Santa Amélia, com 284ha de área e população de 254 índios, sendo a aldeia conhecida como Sede. Nessa aldeia, há predomínio de guaranis e fica a $5 \mathrm{~km}$ da cidade; e

e) Pinhalzinho, que pertence ao Município de Tomazina, com 593ha e população de 99 índios. Sua aldeia chama-se Sede. Nessa aldeia, também predomina a população guarani e dista $7 \mathrm{~km}$ da cidade.

Apesar de haver predomínio de uma determinada etnia em cada aldeia, observa-se também miscigenação, com alta proporção de mestiços em todas elas.

Todas as aldeias são próximas às cidades a que pertencem (de 3 a $30 \mathrm{~km}$ ) e possuem fácil acesso, pois as estradas apresentam boas condições, facilitando o transporte por ônibus e caronas ou deslocamentos a pé.

Um outro ponto a ser levantado é que os modos de vida desses povos são muito semelhantes entre si, inclusive do ponto de vista econômico. Cultivam produtos de subsistência (arroz, milho e feijão) e deslocam-se com muita freqüência para centros urbanos para comercializar artesanatos, orquídeas e outros produtos naturais.

Para a construção dos indicadores, trabalhou-se com a totalidade dos óbitos e nascimentos vivos de índios de cada reserva indígena, no período de 1990 a 1999 . Os dados foram levantados em livros de registros de nascimentos e óbitos existentes em cada reserva, bem como em declarações de nascidos vivos e declarações de óbitos para os que nasceram ou morreram em hospitais. Os relatórios enviados à Administração Executiva Regional da 
Fundação Nacional do Índio (FUNAI), em Londrina, também foram analisados, para evitar perda ou duplicidade de dados. A necessidade desse levantamento, em fontes diversas, decorre da não obrigatoriedade do registro, em cartórios, de eventos vitais ocorridos entre indígenas.

Para avaliação da mortalidade indígena, nesse período, foram utilizados os seguintes indicadores: coeficiente de mortalidade infantil e seus componentes (mortalidade neonatal e pós-neonatal), indicador de Swaroop-Uemura (mortalidade proporcional de 50 anos ou mais) e indicador de Guedes adaptado por Pereira. ${ }^{4}$

$O$ coeficiente de mortalidade infantil é considerado um indicador sensível, sendo muito utilizado por refletir as desigualdades sociais. ${ }^{4-6}$ Tradicionalmente, esse coeficiente é subdividido em período neonatal, no qual estão incluídos os óbitos de menores de 28 dias (até 27 dias) e período pós-neonatal, que corresponde aos óbitos ocorridos no período, que vai de 28 dias até um ano. Geralmente, as causas de óbito no período neonatal estão mais relacionadas a problemas decorrentes da gestação e parto, enquanto que, no pós-neonatal, predominam causas relacionadas às condições de vida $\mathrm{e}$ meio ambiente. ${ }^{4,5}$

O indicador de Swaroop-Uemura é uma das formas utilizadas para medir o nível de saúde de uma região, que trabalha com lógica inversa à do coeficiente de mortalidade infantil: quanto maior o índice, melhor o nível de saúde. ${ }^{4}$ As mudanças ocorridas na sociedade, em geral, têm revelado a necessidade de se buscarem novos indicadores que trabalhem, por exemplo, com a proporção de óbitos com idade acima de 65 anos. $^{7}$ No presente estudo, o indicador de Swaroop-Uemura pôde ser utilizado sem prejuízo da análise.

Para uma melhor interpretação do indicador de Guedes, que trabalha com valores que variam de menos 40 a 50 , Pereira $^{4}$ sugeriu uma modificação que permitiu fazê-lo oscilar entre "menos $1 \mathrm{e}$ 1 ", e, quanto mais próximo a "menos 1", pior o nível de saúde; e, quanto mais próximo a 1 , melhor o nível de saúde.
Os indicadores foram apresentados em tabelas, sendo calculados para dois períodos de cinco anos (1990-94 e 199599), a fim de evitar variações aleatórias, e foi analisada a variação percentual, usando como base o primeiro período. $\mathrm{O}$ tamanho da amostra (pequeno) impediu a análise específica por grupos ou terras indígenas.

\section{Resultados}

Para o coeficiente de mortalidade infantil, observou-se uma piora no segundo período (de 57 para 79,9 por mil nascidos vivos). Ao se decompor o coeficiente de mortalidade infantil em coeficientes neonatal e pós-neonatal, nota-se que o componente neonatal foi o que mais contribuiu para o aumento do coeficiente de mortalidade infantil (aumento de 159\%). O componente pós-neonatal, ainda que em menor proporção, também teve sua parcela de contribuição para a piora do coeficiente de mortalidade infantil, com aumento da taxa em torno de $10 \%$ (Tabela 1 ).

Observou-se, ainda, uma diminuição do valor do indicador de SwaroopUemura, revelando, também, uma piora do nível de saúde no segundo período. Esses resultados também são reforçados pelo indicador de Guedes adaptado por Pereira, o qual passa de um valor positivo, no primeiro período, para um valor negativo, no segundo (Tabela 1).

As faixas etárias que contribuíram para a queda do indicador de SwaroopUemura (Tabela 2) foram, respectivamente, a de menores de 28 dias (aumento de $234,2 \%$ ), a de 28 dias a um ano (incremento de $41,4 \%$ ) e a de 20 a 49 anos (aumento de 35,6\%).

Nos períodos em estudo, mesmo com altas proporções de mortalidade por causas mal definidas (cerca de 23\%), foi possível observar um aumento da mortalidade proporcional por causas externas: de sete óbitos no primeiro período para 12 óbitos no segundo, ou seja, de 9 para $15 \%$ do total de óbitos. Esses foram devidos, principalmente, a agressões (três e quatro mortes, no primeiro e segundo período respectivamente) e a acidentes de transporte terrestre (um e quatro óbitos). 
Tabela 1 - Indicadores de saúde e respectivas variações percentuais da população indígena do norte do Paraná nos períodos de 1990 a 1994 e 1995 a 1999

\begin{tabular}{lccc}
\hline Indicadores & $\mathbf{1 9 9 0 - 9 4}^{*}$ & $\mathbf{1 9 9 5 - 9 9 * *}^{*}$ & Variação (\%) \\
\hline Coeficiente de mortalidade infantil*** & 57,0 & 79,9 & $+42,2$ \\
Coeficiente de mortalidade neonatal ${ }^{* * *}$ & 11,4 & 29,6 & $+159,6$ \\
Coeficiente de mortalidade pós-neonatal*** & 45,6 & 50,3 & $+10,3$ \\
Indicador de Swaroop-Uemura (\%) & 41,8 & 26,6 & $-36,4$ \\
Indicador de Guedes adaptado por Pereira ${ }^{4}$ & $+0,12$ & $-0,27$ & $-325,0$ \\
\hline
\end{tabular}

* Número de óbitos: vide Tabela 2. Total de nascidos vivos: 263.

** Número de óbitos: vide Tabela 2. Total de nascidos vivos: 338 .

*** Por mil nascidos vivos.

Tabela 2 - Mortalidade proporcional por faixa etária e respectivas variações percentuais da população indígena do norte do Paraná nos períodos de 1990 a 1994 e 1995 a 1999

\begin{tabular}{|c|c|c|c|c|c|}
\hline \multirow{3}{*}{ Faixa etária } & \multicolumn{4}{|c|}{ Mortalidade proporcional } & \multirow{3}{*}{ Variação (\%) } \\
\hline & \multicolumn{2}{|c|}{$1990-94$} & \multicolumn{2}{|c|}{ 1995-99 } & \\
\hline & № & $\%$ & № & $\%$ & \\
\hline 0 a 28 dias & 3 & 3,8 & 10 & 12,7 & $+234,2$ \\
\hline 28 dias a 1 ano & 12 & 15,2 & 17 & 21,5 & $+41,4$ \\
\hline 1 a 4 anos & 11 & 13,9 & 6 & 7,6 & $-45,3$ \\
\hline 5 a 19 anos & 6 & 7,6 & 6 & 7,6 & - \\
\hline 20 a 49 anos & 14 & 17,7 & 19 & 24,0 & $+35,6$ \\
\hline 50 anos ou mais & 33 & 41,8 & 21 & 26,6 & $-36,4$ \\
\hline Total & 79 & 100,0 & 79 & 100,0 & - \\
\hline
\end{tabular}

\section{Discussão}

A comparação entre os dois períodos denunciou uma piora do nível de saúde indígena do norte do Paraná nos últimos anos.

Segundo Pereira, ${ }^{4}$ o coeficiente de mortalidade infantil é um dos indicadores mais utilizados para medir o nível de saúde e desenvolvimento de uma região. Quando se observa diminuição desse coeficiente, pode-se pensar numa possível queda da mortalidade por doenças diarréicas, expansão da rede pública assistencial e aumento de sua cobertura. ${ }^{8}$

O coeficiente de mortalidade infantil entre indígenas do norte do Paraná, que já era alto no primeiro período, apresentou piora no segundo, atingindo o valor de 79,9 por mil nascidos vivos, uma taxa próxima à verificada para o Brasil no início da década de $80 .{ }^{6}$ Em 1995, o município de Londrina apresentou um coeficiente de mortalidade infantil de $15,2^{9}$ e o Brasil de 37,7 por mil nascidos vivos. ${ }^{10}$ A taxa de mortalidade infantil encontrada para o período de 1995-99, em relação à população do presente estudo, foi maior ainda que a da população indígena da Colômbia no ano de 1990 , estimada em 63,3 por mil nascidos vivos. ${ }^{11}$

A mortalidade é distribuída de forma desigual pelos períodos de vida; dessa forma, quando se avalia o componente neonatal, verifica-se que este é o maior responsável pelo aumento do coeficiente de mortalidade infantil dos indígenas, pois teve uma variação de $159,6 \%$, do 
primeiro para o segundo período. Ainda assim, observa-se que o componente pósneonatal ainda é o principal responsável pela mortalidade infantil indígena no norte do Paraná, indicando condições precárias de vida, como sugerido por alguns autores. ${ }^{4-8}$

A mortalidade neonatal sofre influência de dois grupos de fatores: condições de vida e qualidade e acesso à assistência à saúde. ${ }^{12}$ No período analisado, segundo informações de técnicos da FUNAI, não houve alteração no padrão de assistência à saúde dos grupos indígenas do norte do Paraná. Assim, pressupõe-se que o aumento do coeficiente de mortalidade neonatal, no período de 1995-99, esteja mais relacionado à piora nas condições de vida.

A mortalidade infantil pós-neonatal sofreu um acréscimo de $10 \%$ entre os períodos em estudo, o que indica, também, queda dos indicadores sociais. Esse aumento do componente pós-neonatal nos obriga a refletir nas desigualdades sociais que ainda fazem parte de nossa realidade.

Atualmente, algumas pesquisas têm demonstrado melhoras dos indicadores de saúde, com melhor acesso a serviços de saúde, nutrição, educação e saneamento básico. ${ }^{10,13,14}$ Isso não reflete, infelizmente, a realidade de todas as regiões ou populações, fato relatado também em um estudo de coorte no Sul do Brasil, onde se pôde observar importantes desigualdades sociais, apesar da queda da mortalidade infantil. ${ }^{15} \mathrm{O}$ aumento da mortalidade infantil pós-neonatal na população indígena do norte do Paraná corrobora esses resultados, ou seja, o declínio da mortalidade não é homogêneo. Cabe destacar que a cidade do Recife, no ano de 1969, praticamente trinta anos antes, apresentava um coeficiente de mortalidade infantil pós-neonatal de 55,9 por mil nascidos vivos, ${ }^{4}$ valor inferior ao encontrado neste estudo em ambos os períodos.

$\mathrm{O}$ indicador de Swaroop-Uemura demonstra um aumento da mortalidade precoce no segundo período, pois mais de $70 \%$ dos óbitos ocorreram antes dos
50 anos. No ano de 1994, a cidade de Londrina apresentou uma mortalidade proporcional em pessoas acima de 50 anos de $67,1 \%,{ }^{10}$ e essa informação reforça o que foi encontrado neste estudo, ou seja, que o estado de saúde da população indígena é muito mais precário.

Apesar da dificuldade de se trabalhar nesse grupo populacional com mortalidade proporcional por causas, devido ao grande número de óbitos por causas mal definidas, um aspecto que se deve salientar é a crescente mortalidade por causas externas, resultante, muitas vezes, de conflitos interétnicos e abuso de álcool, que trazem graves conseqüências tanto para crianças como para adultos e interferem diretamente nos anos produtivos de uma comunidade. ${ }^{16}$

No presente estudo, observou-se aumento da mortalidade proporcional por causas externas, decorrente principalmente de acidentes de transporte terrestre, fato que pode ser explicado pela grande presença de índios às margens de importantes rodovias para a venda de produtos artesanais. Essas mortes, em geral prematuras, trazem reflexos diretos na piora no indicador de Swaroop-Uemura e no de Guedes adaptado por Pereira. ${ }^{4}$

$\mathrm{O}$ indicador de Guedes adaptado por Pereira, corrobora, portanto, os indicadores anteriores quanto à piora do nível de saúde da população indígena no período de 1995 a 1999 e chama a atenção por ser um valor pior que o apresentado no Distrito Federal em 1980 (menos 0,11 ), que já era considerado um nível baixo de saúde. ${ }^{4}$ Alguns pesquisadores ${ }^{17,18}$ discutem que, em algumas situações, o tratamento dado à população indígena é diferenciado daquele prestado ao restante da população, sendo aquele inferior em qualidade e quantidade de serviços ofertados, refletindo desigualdades que ainda existem e que se traduzem em piores valores de indicadores tradicionais de saúde.

Quando a mortalidade é analisada, discriminando as faixas etárias, observouse que o aumento da mortalidade precoce ocorreu, principalmente, devido a mortes 
de menores de um ano e de 20 a 49 anos, provavelmente devido à piora das condições de vida, além de, possivelmente, problemas relacionados ao alcoolismo e à violência decorrentes da atual situação de vida dos indígenas, que os colocam em contato com a sociedade envolvente sem, no entanto, propiciar condições para uma vida digna e segura. Esses fatos começam, atualmente, a ganhar espaço na imprensa leiga da região, como demonstram matérias encabeçadas pelos seguintes títulos: "Índios vivem em situação de miséria", ${ }^{19}$ "Crianças viram catadoras de papel" [crianças indígenas] ${ }^{20}$ e "Crianças caingangues mendigam nos semáforos". ${ }^{21}$

Este trabalho demonstra, assim, que essa realidade cruel também se reflete em valores desfavoráveis de indicadores de saúde, muito piores do que a média da região e do país, o que faz pressupor a necessidade premente de ações coordenadas e intersetoriais que visem à melhoria do nível de saúde e de vida dessas populações indígenas. Algumas ações concretas poderiam ser sugeridas com a finalidade de colaborar para a reversão desse quadro: ampliação da assistência à saúde, em especial melhoria da atenção à gestação, ao parto e ao recém-nascido; envolvimento de líderes indígenas no planejamento e administração dos distritos sanitários indígenas e, finalmente, a organização das informações em saúde dessas comunidades, a fim de permitir um adequado monitoramento do nível de saúde desses grupos indígenas e da atenção à saúde prestada nesses locais.

\section{Referências bibliográficas}

1. Fundação Nacional de Saúde. Política nacional de atenção à saúde dos povos indígenas. Brasília: Ministério da Saúde; 2000.

2. Conselho Nacional de Saúde. A jornada de uma raça chega ao século 21. Revista da Saúde [série online] 2000 Dez [Capturada em 2002 jan 08];1(1):4-5. Disponível em: http:// conselho.saude.gov.br/biblioteca/ revistas/Resvita_Indigena.pdf.
3. Battistelli E, Saraiva MP. Os povos indígenas do Paraná. Curitiba: Assessoria para Assuntos Indígenas da Casa Civil; 1997.

4. Pereira MG. Epidemiologia: teoria e prática. Rio de Janeiro: GuanabaraKoogan; 1995.

5. Rouquayrol MZ. Epidemiologia e Saúde. $3^{\mathrm{a}}$ ed. Rio de Janeiro: Medsi; 1988.

6. Tavares R, Monteiro MFG. População e condições de vida. In: Guimarães R, Tavares RAW, organizadores. Saúde e sociedade no Brasil: anos 80. Rio de Janeiro: Relume Dumará; 1994. p.43-73.

7. Kerr-Pontes LRS, Rouquayrol MZ. A medida da saúde coletiva. In: Rouquayrol MZ, Almeida Filho N. Epidemiologia e Saúde. $5^{\text {a }}$ ed. Rio de Janeiro: Medsi; 1999. p.31-68.

8. Gomes JO, Santos AH. Mortalidade infantil em município da região Centro-Oeste Paulista, Brasil, 19901992. Revista de Saúde Pública 1997;31:330-341.

9. Autarquia do Serviço Municipal de Saúde. Rumos da saúde para Londrina: plano municipal para o biênio 1996-1997. Londrina: Prefeitura do Município de Londrina; 1996.

10. Monteiro CA, Benício MHD, Freitas ICM. Melhorias em indicadores de saúde associados à pobreza no Brasil dos anos 90: descrição, causas e impacto sobre desigualdades regionais. São Paulo: Nupens/USP; 1997.

11. Piñeros-Petersen MC, Ruiz-Salguero M. Aspectos demográficos en comunidades indígenas de tres regiones de Colombia. Salud Pública de México 1998;40:324-329.

12. Lee KS, Paneth N, Gartner LM, Pearlman MA, Gruss L. Neonatal mortality: an analysis of the recent improvement in the United States. American Journal of Public Health 1980;70:15-21.

13. Monteiro CA, Nazário CL. Evolução de condicionantes ambientais da saúde na infância na cidade de São

\section{Este trabalho demonstra valores desfavoráveis de indicadores de saúde, piores do que a média da região e do país.}


Paulo (1984-1996). Revista de Saúde Pública 2000;34:13-18.

14. Monteiro CA, Freitas ICM. Evolução de condicionantes socioeconômicos da saúde na infância na cidade de São Paulo (1984-1996). Revista de Saúde Pública 2000;34(Supl.6):8-12.

15. Menezes AMB, Victora CG, Barros FC, Albernaz E, Menezes FS, Jannke HA et al. Mortalidade infantil em duas coortes de base populacional no Sul do Brasil: tendências e diferenças. Cadernos de Saúde Pública 1996;12 (Supl.1):79-86.

16. Organização Panamericana da Saúde. Salud de los pueblos indigenas en la region de las Americas. Washington, D.C.: OPS; 1993. Documento de trabajo.
17. Telarolli Júnior $\mathrm{R}$, Carvalho $\mathrm{F}$. Algumas considerações sobre a assistência à saúde das populações indígenas. Saúde em Debate 1994; 42:49-53.

18. Langdon SJ. Saúde indígena: a lógica do processo de tratamento. Saúde em Debate 1988; (ed. especial):12-15.

19. Lombardo A. Índios vivem em situação de miséria. Folha de Londrina 2001 Abr 19; Seção Folha do Paraná:7.

20. Augusto L. Crianças viram catadoras de papel. Folha de Londrina 2001 abr 19; Seção Folha do Paraná:7.

21. Barbosa M. Crianças caingangues mendigam nos semáforos. Folha de Londrina 2001 Abr 18; Seção Folha do Paraná:5. 